\title{
An Experimentally Validated Immersed Boundary Model of Fluid-Biofilm Interaction
}

\author{
Garret Dan Vo ${ }^{\mathrm{a}}$, Eric Brindle ${ }^{\mathrm{b}}$, and Jeffrey Heys, ${ }^{\mathrm{c}, *}$ \\ ${ }^{a}$ Department of Mathematics and Physics \\ ${ }^{\mathrm{b}}$ Department of Mechanical Engineering \\ ${ }^{\mathrm{c}}$ Department of Chemical and Biological Engineering \\ Montana State University- Bozeman \\ Bozeman, Montana, 59717, USA \\ *Corresponding author: jeff.heys@gmail.com
}

\begin{abstract}
Biofilms are colonies of microorganisms that live on wetted surfaces in a matrix consisting of polysaccharides, proteins, and nucleic acids. According to the National Institute of Health (NIH), biofilms play a role in over 80 percent of microbial infections in the body and these infections are remarkably difficult to treat with antimicrobial compounds. The objective here is to understand and predict the physical interaction between a biofilm and the surrounding fluid flow. We have developed a biofilm-fluid interaction model, based on the Immersed Boundary Method, to simulate the interaction between the biofilm and a moving fluid. The model predictions of biofilm deformation quantitatively agree with experimental measurements for a range a biofilms using a simple immersed elastic solid to model the biofilm matrix. An immersed viscoelastic solid model is also developed and compared with experimental measurements. The results show that the viscoelastic behavior inherent to the immersed boundary method (even when using a simple immersed elastic solid) is sufficient for some biofilms, but a slightly viscoelastic solid gives more general agreement with experimental measurements.
\end{abstract}

Keywords: biofilm, mathematical model, fluid-structure interaction, immersed boundary method 


\section{Introduction}

Bacterial biofilms reside on most wetted surfaces and form after microbial cells attach to the surface. They are complex mixtures of microorganisms in an extracellular matrix of polysaccharides, proteins and nucleus acids. Because of their unique properties, biofilms play a critical role in engineering and medicine. For example, biofilms cause energy loss in piping systems (Characklis, 1973; Picologlou et al., 1980), increase drag forces on ships (Cooksey and Wigglesworthcooksey, 1995; Schultz and Swain, 2000), contribute to persistent infections (Costerton, 1999), contaminate indwelling catheters (Bortz et al., 2008), promote tooth decay (Furiga et al., 2008), and play a critical role chronic wounds (James et al., 2008). In addition to the problems, damage, and disease caused by biofilms, they also have wide range of beneficial applications, including $\mathrm{CO}_{2}$ sequestration (Mitchell et al., 2008), bioremediation, and enhanced oil recovery (Davis et al., 2006). Due to the important role of biofilms, experimental and theoretical research on biofilms has rapidly increased over the past two decades.

Most of the previously developed models of biofilms have focused on diffusion and transport within the biofilm and were developed to predict their behavior (e.g., microbial death from an antimicrobial agent or the local growth) on the time scale of hours, days, or even weeks (Stewart and Raquepas, 1995; Stewart, 1996; Chen and Kojouharov, 1999; Dodds et al., 2000; Stewart, 2003; Hunt et al., 2005; Duddu et al., 2008). Many critical biofilm phenomena, however, occur over a few seconds or minutes and involve interaction with a flowing fluid (Klapper et al., 2002). For example, biofilm detachments due to an increase in fluid shear or changes in permeability due to fluid flow are not well understood theoretically due to limited fluid-structure interaction models. A recent two-dimensional model based on the eXtended Finite Element Method (XFEM) and the level set method demonstrated erosion due to the interfacial shear stress at the biofilm-fluid interface (Duddu et al., 2009). Two immersed boundary type models have been developed previously to study biofilm-fluid interaction. Dillon et al. (1996; 2000) studied microbe transport through an idealized (cylindrical) two-dimensional porous media with discrete bacterial cells, which could be planktonic, attached to a solid surface, or attached to another cell. A second immersed boundary type model was developed by Alpkvist and Klapper (2007). This model is three-dimensional, and if the springs are stretched beyond a specified distance, they can break resulting in particle (roughly, bacterial) detachment from the biofilm. Neither of the previously development immersed boundary models for biofilms have been experimentally validated or even quantitatively compared with experimental measurements and neither of the models include a viscoelastic description of the biofilm, which has been shown experimentally to be important (Towler et al., 2003).

In this paper, we investigate a fluid-biofilm mechanical interaction model based on the immersed boundary method originally developed to model blood flow (Peskin, 1977). The objective of the model is to predict biofilm deformation on the time scale of seconds in response 
to a relatively rapid change in fluid shear. In the first part of the paper, the model equations for simulating fluid-biofilm interaction are developed to predict deformation, and then the model is compared to experimental measurements. Finally, we study role of the viscoelasticity in the model and compare our result to experimental measurements.

\section{Methods}

The equations of motion for a three-dimensional Newtonian, incompressible fluid are:

$$
\begin{gathered}
\rho\left(\frac{\partial v}{\partial t}+\boldsymbol{v} \cdot \nabla \boldsymbol{v}\right)=-\nabla p+\mu \nabla^{2} \boldsymbol{v}+\boldsymbol{f} \\
\nabla \cdot \boldsymbol{v}=0
\end{gathered}
$$

where $\boldsymbol{v}$ and $p$ are the velocity and pressure of the fluid, respectively. A three-dimensional solid representing the biofilm is then 'immersed' in the fluid and coupled to the fluid through a force balance and a velocity matching condition. This approach requires that the material being represented by the immersed solid (i.e., biofilm) have a density close to the fluid density.

The applied body force per unit volume on the fluid, $\boldsymbol{f}$, is related to the force density in the immersed solid, $\boldsymbol{F}$, by

$$
\boldsymbol{f}(\boldsymbol{x}, \boldsymbol{t})=\int \boldsymbol{F}(q, r, s, t) \cdot \boldsymbol{\delta}(\boldsymbol{x}-\boldsymbol{X}(q, r, s, t)) d q d r d s
$$

where $\boldsymbol{\delta}$ is the three-dimensional Dirac delta function, $\boldsymbol{x}=(x, y, z)$ are the fixed Cartesian coordinates (used for the fluid), $\boldsymbol{X}(q, r, s, t)$ represents the three-dimensional, transient immersed solid in the three-dimensional Cartesian space, and $q, r$, and $s$ are curvilinear coordinates attached to the material.

The immersed solid force density, $\boldsymbol{F}$, in equation (3) is the Fréchet derivative of the elastic energy function, $\boldsymbol{E}(\boldsymbol{X})$, and it is given by:

$$
\boldsymbol{F}_{E}=-\frac{\partial E}{\partial \boldsymbol{X}}
$$

where $\boldsymbol{X}(q, r, s, t)$ is the location of the immersed solid. In addition to balancing the forces between the fluid and immersed solid, the velocity of the fluid must be equal to the velocity of the solid. This condition is imposed by:

$$
\frac{\partial X(q, r, s, t)}{\partial t}=\int \boldsymbol{v}(\boldsymbol{x}, t) \cdot \boldsymbol{\delta}(\boldsymbol{x}-\boldsymbol{X}(q, r, s, t)) \boldsymbol{d x}
$$


Equations (1) - (5) are the standard set of equations for the immersed boundary method and many of its variants. A formal second-order method is used to solve the system of equations (Lai and Peskin, 2000; Heys et al., 2008; Kim and Peskin, 2009). The derivatives in these equations are calculated using a second-order finite difference approximation, and equations (1) and (2) are solved using the Fast Fourier Transform (FFT) method (Peskin, 1982). A consequence of using a FFT solver is that all boundary conditions are periodic. A twodimensional solid surface is immersed in the fluid to represent walls in the domain, and an inflow velocity boundary condition is set by applying an external force per unit volume on the fluid that is equal to the desired velocity (Zhu and Peskin, 2002).

The model domain is a $0.9 \mathrm{~mm} \times 0.9 \mathrm{~mm} \times 1.8 \mathrm{~mm}$ cuboid; chosen to be consistent with the geometry of the validation experiments described below. The shape of the biofilm is based on the shape of experimentally observed biofilms, and the height is $180 \mu \mathrm{m}$, consistent with experimental measurements. The discretization of equations (1) and (2) uses a $16 \times 16$ x 32 grid for the results shown here, but a $32 \times 32 \times 64$ fluid grid (and appropriately refined immersed solid) was also used to ensure that the fluid field was sufficiently resolved with the normal grid. The approximate solution changed by less than $5 \%$ (using an $\mathrm{L}^{2}$ norm) when using the finer grid, so the normal grid was deemed sufficient for the simulations utilized here. The points representing the biofilm (i.e., the solid) are initially spaced so that the maximum distance between points is approximately one-third the distance between fluid grid points. The result is that there are negligible convective flows within biofilm (i.e., the biofilm is practically impermeable) in the rest position (Peskin, 2002). As the biofilm deforms, some of the solid points are spread apart, and, once the spacing between the solid points approaches the spacing between the fluid grid points, the biofilm becomes slightly permeable. To model a biofilm that is permeable, points representing the biofilm should be spaced so that they are roughly the same distance apart as the fluid nodes. Larger spacing leads to greater permeability.

\section{Solid Model}

With regards to modeling a biofilm in fluid, everything in equations (1)-(5) is known with one important exception. The viscosity and density of water are known, and biofilms can be imaged from two different directions (e.g., from the side and above or below) to estimate the three-dimensional initial (or rest) shape of the biofilm (light transmission can also give a very rough approximation of changes in biofilm thickness). The only unknown that remains is the elastic energy function, $\boldsymbol{E}(\boldsymbol{X})$ in equation (4). Typically, the elastic energy is assumed to be of the form

$$
\boldsymbol{E}=\int \boldsymbol{\varepsilon}\left(\left|\frac{\partial X}{\partial s}\right|\right) d q d r d s
$$


where the local energy density, $\boldsymbol{\varepsilon}$, is a function of the strain (Peskin, 2002). The results in this paper utilize two different elastic energy functions. The first elastic energy function models the solid as a collection of 1-D springs woven to form a three-dimensional volume. This assumption results in the following form for the energy function for each 1-D spring:

$$
\boldsymbol{E}(\boldsymbol{X})=\frac{1}{2} c_{s} \int\left(\left|\frac{\partial \boldsymbol{X}}{\partial s}\right|-1\right)^{2} d s
$$

where $c_{S}$ represents the stiffness of the spring.

If a solid body is modeled as a purely elastic material, the model would predict that the material moves instantaneously in response to an applied force. Using equation 6a to model the biofilm as a collection of simple 1-D elastic springs does not result in a material that can move instantaneously in response to an applied fluid force because the solid is coupled to a viscous fluid through a force (and velocity) balance. In this way, the biofilm in this model acts as a viscoelastic material even though it is modeled with simple springs because it is immersed in (and coupled to) a viscous fluid. However, it is possible that the extracellular matrix of a biofilm, even without any fluid, is viscoelastic requiring a viscoelastic solid model (Klapper et al., 2002). To examine this possibility, a second elastic energy function is examined here, which models the solid as a collection of 1-D springs and dashpots in parallel. For the case of a viscoelastic,

Kelvin-Voigt model, the stress-strain relationship is given by $\sigma=E \epsilon+\mu \frac{d \epsilon}{d t}$, and the energy function for each viscoelastic spring-dashpot connection is given by

$$
\boldsymbol{E}(\boldsymbol{X})=\frac{1}{2} c_{s} \int\left(\left|\frac{\partial \boldsymbol{X}}{\partial s}\right|-1\right)^{2} d s+\frac{1}{2} c_{v i s} \frac{d}{d t}\left(\int\left(\left|\frac{\partial X}{\partial s}\right|-1\right)^{2} d s\right)
$$

where $c_{v i s}$ represents the magnitude of the viscous dampening in the solid. The one-dimensional viscoelastic springs are again woven into a three-dimensional solid representing the biofilm.

\section{Validation Experiments}

In order to compare the biofilm model given by equations (1)-(6) to the mechanical behavior of an actual biofilm, a simple flow cell experiment was conducted (Fig. 1). An initial culture was started for inoculation using full strength tryptic soy broth (TSB) with Staphylococcus epidermis (ATCC 35984). The S. epidermidis inoculum incubated for 24 hours on a shaker plate at $37^{\circ} \mathrm{C}$. The 24 hour culture was then used to inoculate a square glass capillary flow cell reactor $(0.9 \mathrm{~mm}$ I.D.) for two hours at a low flow rate (less than $1 \mathrm{~mL} / \mathrm{min}$ of 10 percent TSB). The glass capillary now contained mature biofilm clusters, and, because the channel was glass, the biofilm can be observed using a microscope and camera (Nikon Eclipse E800 microscope, Hamamatsu C2400-75AH camera and MetaVue software). Once mature biofilm clusters have been established, the flow in the channel is rapidly increased to $10 \mathrm{~mL} / \mathrm{min}$ 
using a continuous cycle syringe pump, which results in a Reynolds number of approximately 230 based on channel size, for 15 seconds causing the biofilm to deform due to the fluid stress. Finally, the flow in the channel is stopped; allowing the biofilm to return to a stress free state. Throughout the experiment, the biofilm is imaged for a total of 30 seconds to capture both elongation and relaxation of a particular biofilm cluster. A detailed description of the experimental protocol is given in (Brindle, 2009).

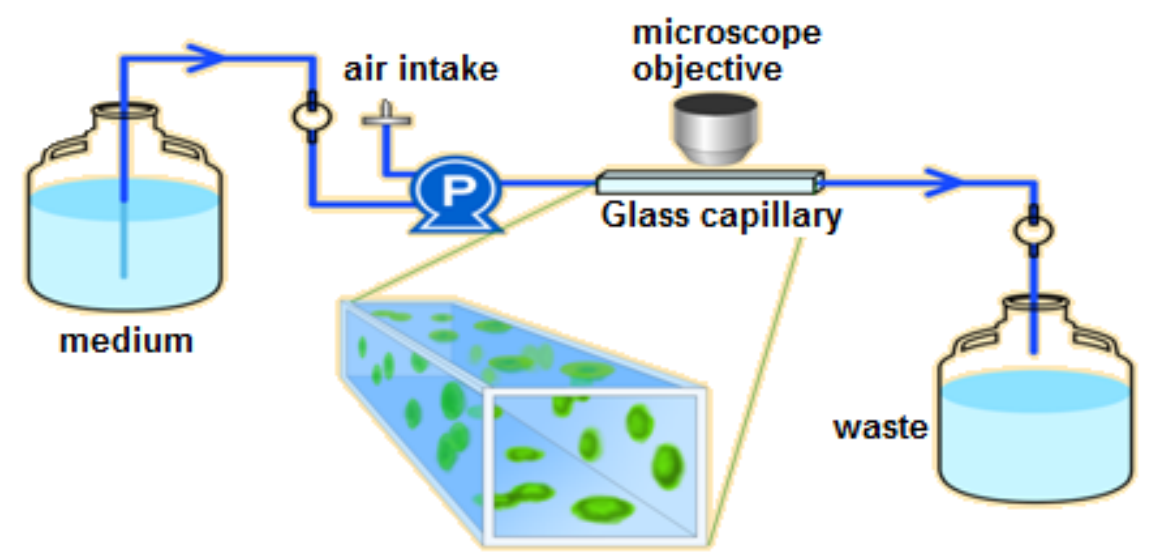

Figure 1. Setup for capillary flow cell reactor during growth of S. epidermidis biofilm

\section{Results}

The deformation of a typical biofilm cluster is shown at rest and under fluid flow in Fig. $2 \mathrm{a}$ and $2 \mathrm{~b}$, respectively. The flow is from left to right, and the biofilm is displaced in that direction by the shearing forces from the fluid. This behavior can be replicated using the model described here, and the model predictions are shown in Fig. 2c (no flow) and 2d (with flow). For this 3-dimensional simulation, $c_{s}=1 \times 10^{-5} \frac{\mathrm{g}}{\mathrm{mm}^{2} \mathrm{~s}^{2}}$ was used. 
(a)

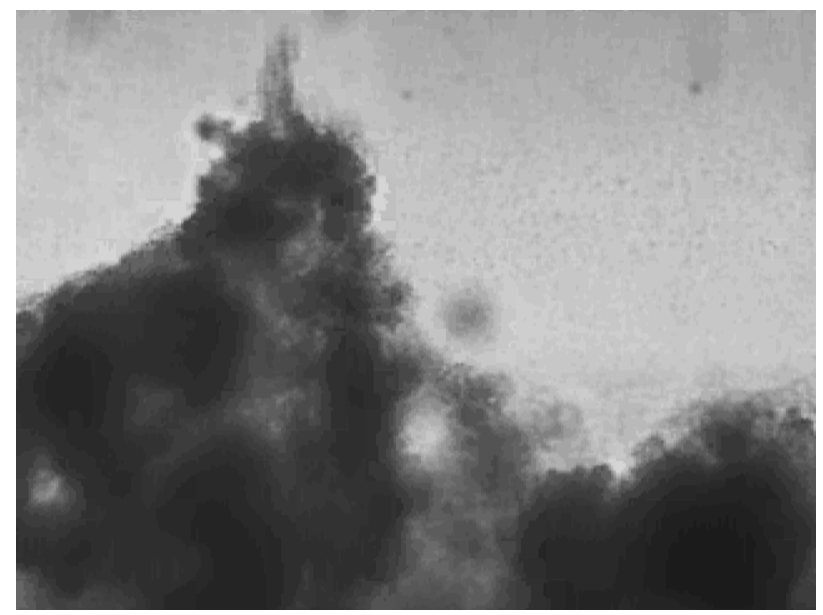

(c)

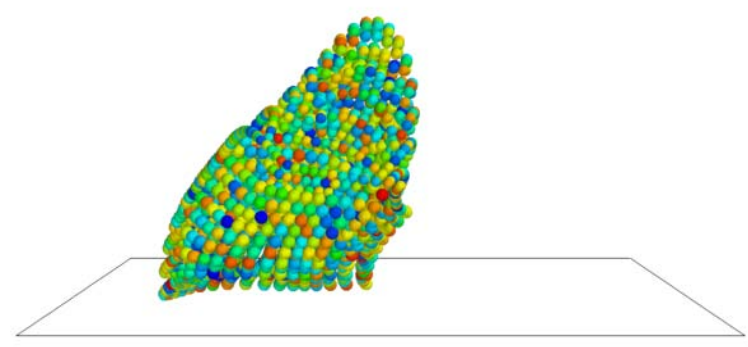

(b)

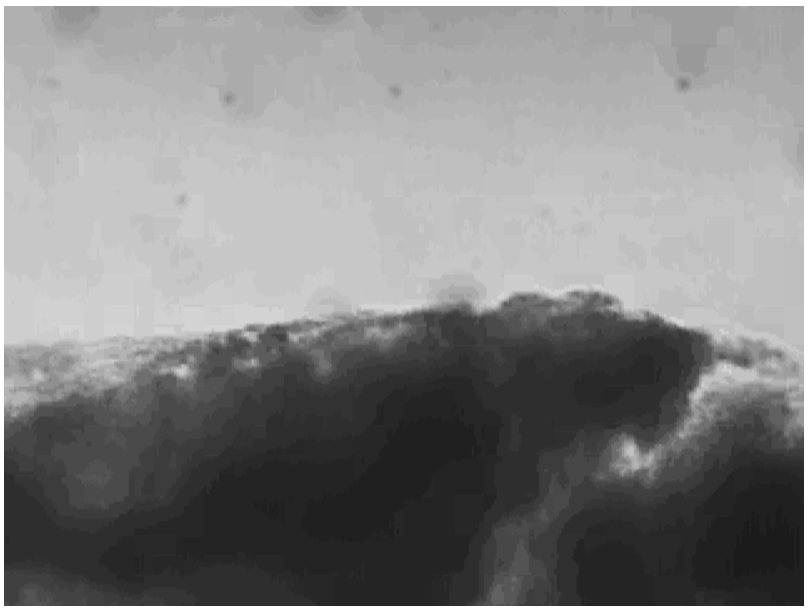

(d)

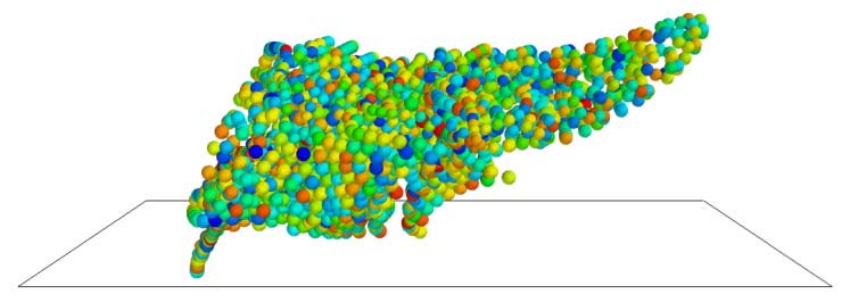

Figure 2. (a) A biofilm grown in a flow cell and (b) the same biofilm being deformed by flow. (c) A three-dimensional simulation of a biofilm before flow and (d) being deformed by a flow of the same magnitude as the experimental flow cell.

For a more quantitatively comparison of the experimental biofilm deformation to the model predictions, we calculated an approximate strain using a fixed fiducial point near the front attachment point of the biofilm and a second, moving fiducial point at the peak of the biofilm (Brindle, 2009). The range of measured strains for the experimental results ( $\mathrm{n}=8$ biofilms) are indicated by the shaded box in Fig. 3, and the experimental mean is indicated by the dashed line. The strains predicted by the model for different values of $\boldsymbol{c}_{\boldsymbol{s}}$ are also shown on Fig. 3 by the square points. These results indicate that $c_{s}=5 \times 10^{-5} \frac{\mathrm{g}}{\mathrm{mm}^{2} \mathrm{~s}^{2}}$ gives the best agreement between the model prediction and experimental measurement. 


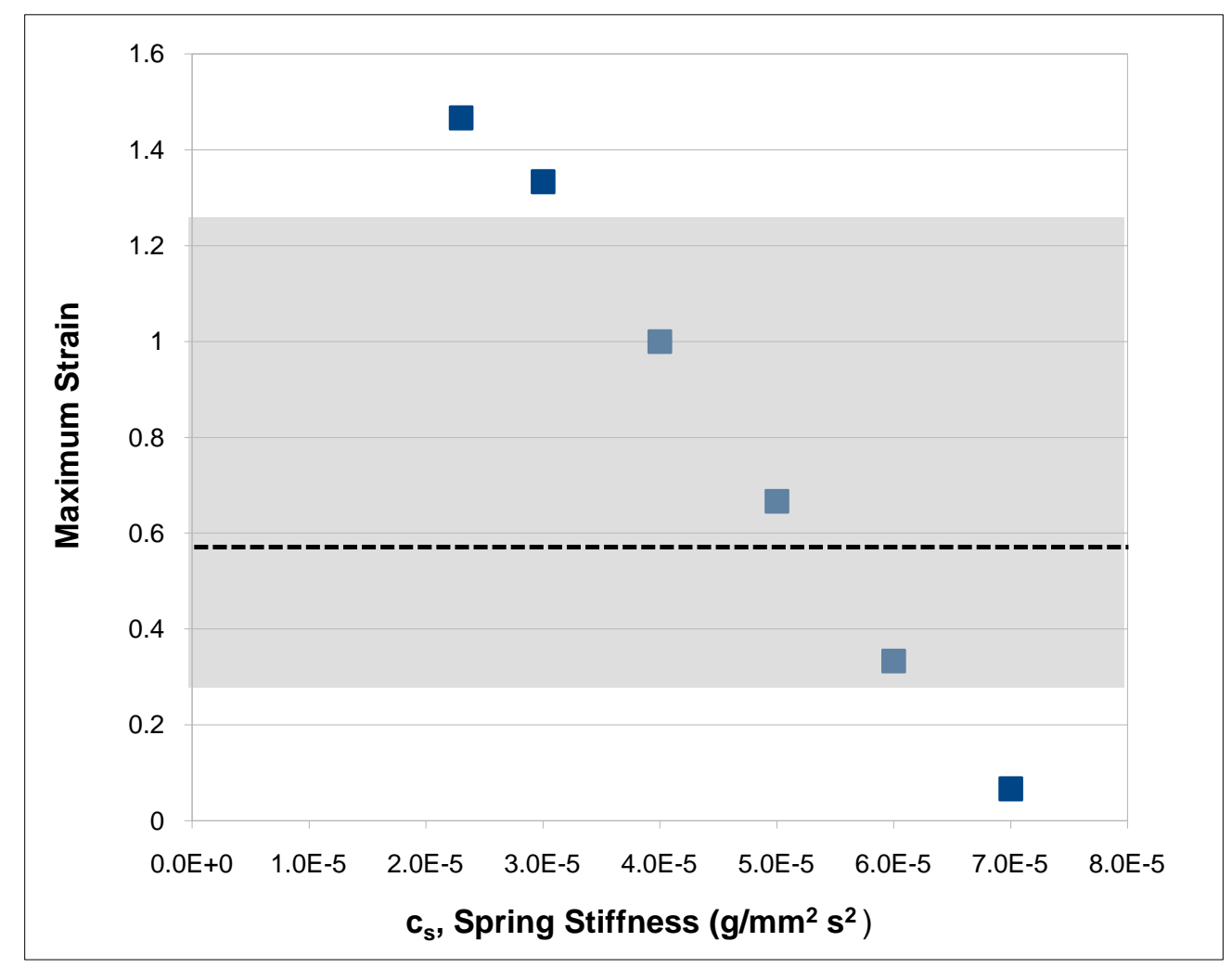

Figure 3. Range of approximate biofilm strains observed experimentally (large, shaded box) and the mean (dashed line), and the model strain for different values of $c_{s}$ (small squares).

One of the outstanding questions in biofilm research is the impact of the soft, flexible biofilm on the flowing fluid. Figure 4 shows a deforming biofilm with streamlines representing the fluid as it travels around the biofilm. The fluid flow is slowest near the solid surface and near the biofilm, and the deflection of fluid around the biofilm causes significantly higher velocities above the biofilm. Depending on the shape of the biofilm and the velocity of the fluid, small recirculations can sometimes be observed at the base of the biofilm on both the upstream and downstream side. These recirculations would have an impact on the delivery of an antimicrobial agent to the biofilm because they would cause most of the antimicrobial to contact the top of the biofilm and limit exposure of the base of the biofilm. 


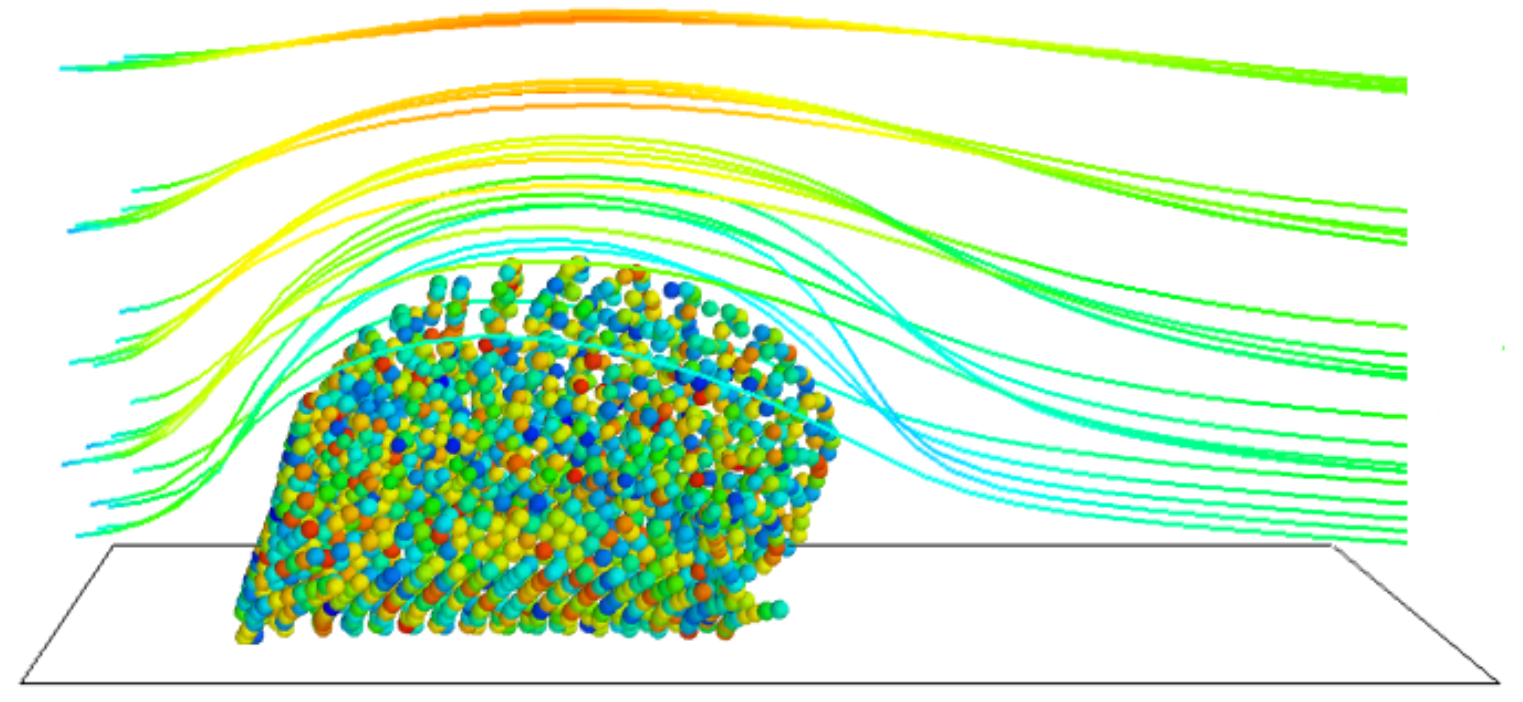

Figure 4. Fluid streamlines for flow moving left to right as it encounters a deforming biofilm.

As stated in the methods section, immersed boundary type methods are inherently viscoelastic because they involve the coupling of a solid to a viscous fluid through a force and velocity matching terms. The solid in the model would deform instantaneously if the fluid was not present, but the viscous fluid in an immersed boundary model prevents instantaneous solid motion even when a purely elastic model is used for the solid. A high viscosity fluid would dampen solid motion more than a low viscosity fluid. The previous results used a purely elastic solid to model the biofilm, and this model accurately captured the magnitude of the solid deformation observed experimentally, but the next set of results is focused on a temporal comparison between the model and experimental measurements. Specifically, the necessity and importance of a nonzero value for $c_{v i s}$ in equation $6 \mathrm{~b}$ is examined.

The first set of simulations and experimental measurements compares the total time for a biofilm to fully deform under a steady fluid shear. Higher viscoelasticity in a Kelvin-Voigt solid (i.e., higher values for $c_{v i s}$ ) will increase the time required for full deformation. It should be noted that using a Kelvin-Voigt solid does not significantly change the shape of the deformation (i.e., the biofilm deformation predicted in Fig. 2 still holds) but only changes the time scale of the deformation (i.e., deformation is slower). Figure 5 shows the range of times required to reach full strain in 8 biofilm experiments (shaded box) and the mean time (dashed line). The time required for the model biofilm is also shown in Fig. 5 using the smaller boxes. For the model to agree well with the experimental measurements, a small value for $c_{v i s}$ (value) is normally required. These results indicate that the viscoelasticity that is inherent in the immersed 
boundary method alone with an elastic solid is nearly sufficient to capture the typically observed deformation times within a biofilm. A small amount of viscoelasticity in the solid, however, is required to correctly capture most of the results observed in the experimental biofilms, including the average result.

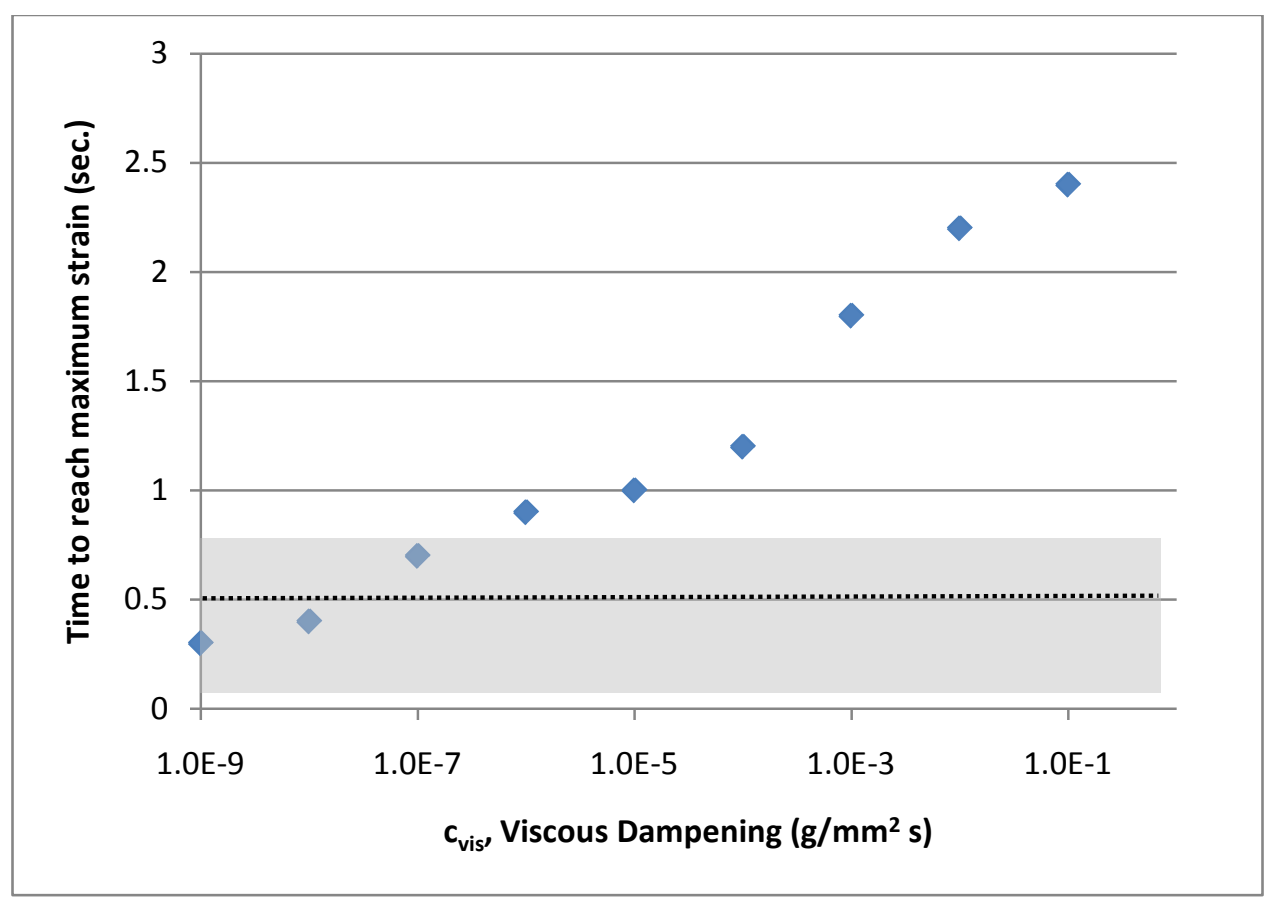

Figure 5: The time required for the biofilm to completely deform during the validation experiments (large grey box) with the mean time also shown (dashed line), and the time for the biofilm in the model to reach full deformation for different values of $c_{v i s}$ (small squares).

Another important quantitative comparison between the model and experimental measurements is to analyze the amount of recovery for the biofilm when the moving fluid flow is stopped and the biofilm returns to an unstrained position. Recovery is defined here as

$$
\text { \%recovery }=\left(1-\frac{\epsilon_{\text {recovery }}}{\epsilon_{\max }}\right) \cdot 100 \%
$$

where $\epsilon_{\max }$ is the maximum strain under fluid shear and $\epsilon_{\text {recovery }}$ is the strain in the biofilm after the flow has stopped. As before, $\in$ is a simple strain based on only two fiducial points, a fixed point and a point at the peak of the biofilm. A recovery of $100 \%$ implies the biofilm fully returns to its initial, unstrained shape once the flow is stopped, and a recovery of $0 \%$ implies that the biofilm deforms under flow but does not return at all towards its initial shape after the flow is stopped. Many types of viscoelastic materials will only partially recovery to their original, no 
flow shape. Table 1 shows the recovery predicted by the model for different values of $c_{s}$. In general, a larger value for $c_{s}$ (i.e., a stiffer spring) corresponds to a larger amount of recovery. The results in the table show that the model prediction is consistent with experimental observations for a variety of different biofilms. Identical results are obtained for non-zero values of $c_{v i s}$, but the viscoelastic solid slows the temporal rate of recovery.

Table 1. Amount of recovery for a biofilm after the flow of a shear fluid is stopped for both the model and experimental measurements.

\begin{tabular}{ll}
\hline $\boldsymbol{c}_{\boldsymbol{s}}$ & Recovery \\
\hline $2.3 \times 10^{5}$ & $53 \%$ \\
$3.0 \times 10^{5}$ & $60 \%$ \\
$4.0 \times 10^{5}$ & $80 \%$ \\
$5.0 \times 10^{5}$ & $85 \%$ \\
$6.0 \times 10^{5}$ & $87 \%$ \\
\hline Experimental biofilm (n=8) & $74 \% \pm 23 \%$ \\
\hline
\end{tabular}

\section{Conclusion}

The biofilm model described here is based on the immersed boundary method, and the biofilm matrix is modeled as a woven collection of one-dimensional springs or Kelvin-Voigt elements (a spring and dashpot in parallel). The model accurately simulates the total deformation of a biofilm under shear due to a moving fluid, showing quantitative agreement with experimental measurements. The model is also able to predict the impact of the biofilm on the fluid as it is deflected by the biofilm. When a viscoelastic solid is used in the model, the time required for the biofilm to fully deform, as predicted by the model, quantitatively agrees with experimental measurements at small values of $c_{v i s}$, the viscous term in the viscoelastic solid model. Finally, the recovery of the biofilm back to its no-flow shape after the fluid flow is stopped is consistent between the model prediction and experimental measurements. Overall, the model was able to accurately predict all aspects of short time scale biofilm behavior observed in the experiments utilized here for the validation of the model. Biofilms exhibit a wide range of mechanical properties, which change with the bacterial strain and the age of the biofilm. The current two parameter model $\left(c_{s}\right.$ and $c_{v i s}$ ) is able to more than capture the range of mechanical properties observed for Staphylococcus epidermidis biofilms by varying the two parameters. The development of a model for other bacterial biofilms will require the determination of the model parameters for those unique biofilms. 


\section{Acknowledgements}

This work was supported by NSF grant CMMI-0849433. The authors wish to thank Dr. Phil Stewart, head of the Center for Biofilm Engineering, for insight and encouragement with this work. 


\section{References}

Alpkvist, E. and I. Klapper (2007). "Description of Mechanical Response Including Detachment Using a Novel Particle Model of Biofilm/Flow Interaction." Wat. Sci. Tech. 55: 265-273.

Bortz, D. M., T. L. Jackson, K. A. Taylor, A. P. Thompson and J. G. Younger (2008). "Klebsiella pneumoniae flocculation dynamics." Bulletin of Mathematical Biology 70(3): 745-768.

Brindle, E. R. (2009). The Effect of Treatments on the Mechanical Properties of Staphylococcus Epidermis Biofilms Under Fluid Shear and Mechanical Indentation. Mechanical Engineering. Bozeman, Montana State University. M.S.: 191.

Characklis, W. G. (1973). "Attached Microbial Growths .1. Attachment and Growth." Water Research 7(8): 1113-1127.

Chen, B. M. and H. V. Kojouharov (1999). "Non-standard numerical methods applied to subsurface biobarrier formation models in porous media." Bulletin of Mathematical Biology 61(4): 779-798.

Cooksey, K. E. and B. Wigglesworthcooksey (1995). "Adhesion of Bacteria and Diatoms to Surfaces in the Sea - a Review." Aquatic Microbial Ecology 9(1): 87-96.

Costerton, J. W. (1999). "Introduction to biofilm." Int J Antimicrob Agents 11(3-4): 217-21; discussion 237-9.

Davis, C. A., E. Atekwana, E. Atekwana, L. D. Slater, S. Rossbach and M. R. Mormile (2006). "Microbial growth and biofilm formation in geologic media is detected with complex conductivity measurements." Geophysical Research Letters 33(18): -.

Dillon, R. and L. Fauci (2000). "A microscale model of bacterial and biofilm dynamics in porous media." Biotechnology and Bioengineering 68(5): 536-547.

Dillon, R., L. Fauci, A. Fogelson and D. Gaver (1996). "Modeling biofilm processes using the immersed boundary method." Journal of Computational Physics 129(1): 57-73.

Dodds, M. G., K. J. Grobe and P. S. Stewart (2000). "Modeling biofilm antimicrobial resistance." Biotechnology and Bioengineering 68(4): 456-465.

Duddu, R., S. Bordas, D. Chopp and B. Moran (2008). "A combined extended finite element and level set method for biofilm growth." International Journal for Numerical Methods in Engineering 74(5): 848-870.

Duddu, R., D. L. Chopp and B. Moran (2009). "A Two-Dimensional Continuum Model of Biofilm Growth Incorporating Fluid Flow and Shear Stress Based Detachment." Biotechnology and Bioengineering 103(1): 92-104.

Furiga, A., M. Dols-Lafargue, A. Heyraud, G. Chambat, A. Lonvaud-Funel and C. Badet (2008). "Effect of antiplaque compounds and mouthrinses on the activity of glucosyltransferases from Streptococcus sobrinus and insoluble glucan production." Oral Microbiology and Immunology 23(5): 391-400.

Heys, J. J., T. Gedeon, B. C. Knott and Y. Kim (2008). "Modeling arthropod filiform hair motion using the penalty immersed boundary method." Journal of Biomechanics 41(5): 977-984.

Hunt, S. M., M. A. Hamilton and P. S. Stewart (2005). "A 3D model of antimicrobial action on biofilms." Water Science and Technology 52(7): 143-148.

James, G. A., E. Swogger, R. Wolcott, E. D. Pulcini, P. Secor, J. Sestrich, J. W. Costerton and P. S. Stewart (2008). "Biofilms in chronic wounds." Wound Repair and Regeneration 16(1): 37-44.

Kim, Y. and C. S. Peskin (2009). "3-D Parachute simulation by the immersed boundary method." Computers \& Fluids 38(6): 1080-1090.

Klapper, I., C. J. Rupp, R. Cargo, B. Purvedorj and P. Stoodley (2002). "Viscoelastic fluid description of bacterial biofilm material properties." Biotechnology and Bioengineering 80(3): 289-296.

Lai, M. C. and C. S. Peskin (2000). "An immersed boundary method with formal second-order accuracy and reduced numerical viscosity." Journal of Computational Physics 160(2): 705-719. 
Mitchell, A. C., A. J. Phillips, M. A. Hamilton, R. Gerlach, W. K. Hollis, J. P. Kaszuba and A. B. Cunningham (2008). "Resilience of planktonic and biofilm cultures to supercritical CO2." Journal of Supercritical Fluids 47(2): 318-325.

Peskin, C. S. (1977). "Numerical-Analysis of Blood-Flow in Heart." Journal of Computational Physics 25(3): 220-252.

Peskin, C. S. (1982). "The Fluid-Dynamics of Heart-Valves - Experimental, Theoretical, and Computational Methods." Annual Review of Fluid Mechanics 14: 235-259.

Peskin, C. S. (2002). "The Immersed Boundary Method." Acta Numerica 11: 479-517.

Picologlou, B. F., N. Zelver and W. G. Characklis (1980). "Biofilm Growth and Hydraulic Performance." Journal of the Hydraulics Division-Asce 106(5): 733-746.

Schultz, M. P. and G. W. Swain (2000). "The influence of biofilms on skin friction drag." Biofouling 15(13): $129-139$.

Stewart, P. S. (1996). "Theoretical aspects of antibiotic diffusion into microbial biofilms." Antimicrobial Agents and Chemotherapy 40(11): 2517-2522.

Stewart, P. S. (2003). "Diffusion in biofilms." Journal of Bacteriology 185(5): 1485-1491.

Stewart, P. S. and J. B. Raquepas (1995). "Implications of Reaction-Diffusion Theory for the Disinfection of Microbial Biofilms by Reactive Antimicrobial Agents." Chemical Engineering Science 50(19): 3099-3104.

Towler, B. W., C. J. Rupp, A. B. Cunningham and P. Stoodley (2003). "Viscoelastic properties of a mixed culture biofilm from rheometer creep analysis." Biofouling 19(5): 279-285.

Zhu, L. D. and C. S. Peskin (2002). "Simulation of a flapping flexible filament in a flowing soap film by the immersed boundary method." Journal of Computational Physics 179(2): 452-468. 